\title{
Avanços e perspectivas da produção científica brasileira em periódicos internacionais na área de comportamento motor
}

\section{Progress and perspectives of the Brazilian scientific production in international journals in the field of motor behavior}

Ana Paula Kogake Claudio Luis Augusto Teixeira

1 Universidade de São Paulo, Escola de Educação Física e Esporte, Laboratório Sistemas Motores Humanos, São Paulo, Brasil.

Recebido em 10/07/08 Revisado em 24/10/08 Aprovado em 13/04/09
Resumo - Considerando-se que um dos principais indicadores de produção científica é a quantidade de artigos publicados em revistas de ampla circulação internacional e que, aparentemente, existe um crescente interesse pela área de comportamento motor, no presente estudo, foi realizado um levantamento de artigos publicados nesta área por pesquisadores brasileiros, nos últimos 10 anos (1999-2008), em periódicos internacionais com conceito "Qualis International-A" e "Qualis International-B" pela CAPES. Esta quantificação foi feita com o propósito de formar um ponto de vista qualificado a respeito do perfil da produção científica brasileira de repercussão internacional em comportamento motor. A identificação dos artigos foi feita por meio dos sistemas de busca Google Acadêmico, Pubmed, Science Direct e Scopus, restringindo-se a busca por termos característicos desta área de estudo e por vínculo a universidades brasileiras. Os resultados indicaram aumento da produção nos 5 anos finais do período, com ápice em 2006. Foi observado, ainda, que a produção tem se concentrado em quatro universidades públicas. Estes resultados sugerem que, para continuar crescendo, os grupos formados mais recentemente deveriam procurar colaboração com laboratórios produtivos, descentralizando a produção científica.

Palavras-chave: Atividade motora; Comportamento; Fator de impacto; Indicadores de produção científica; Sistemas de avaliação das publicações.

Abstract - In view of the fact that one of the key indicators of scientific production is the number of papers published in international journals, and of the apparent growing interest in the area of motor behavior, we conducted a survey of articles published by Brazilian researchers in this area over the last 10 years (1999-2008) in international journals rated "Qualis International-A" and "Qualis International-B" by CAPES. This quantification was performed to provide a qualified viewpoint regarding the profile of Brazilian scientific production of international repercussion in the area of motor behavior. Articles were identified using the Google Scholar, Pubmed, Science Direct, and Scopus search systems, with the search being restricted to characteristic terms involving motor behavior and to researchers associated with Brazilian universities. The results showed an increase in production over the last 5 years of the period studied, with the peak in 2006. In addition, Brazilian scientific production was concentrated in four public universities. These results suggest that in order to keep growing, the new groups should work in collaboration with productive laboratories, decentralizing the scientific production.

Key words: Motor activity; Behavior; Impact factor; Scientific publication indicators; Systems for evaluation of publications. 


\section{INTRODUÇÃO}

O acompanhamento dos recentes eventos científicos específicos sobre comportamento motor-abrangendo as disciplinas de controle motor, aprendizagem motora e desenvolvimento motor - tem tornado aparente o crescente interesse por esta área de estudo. Tal percepção tem sido reforçada pelas várias publicações, em âmbito nacional, na forma de resumos em anais de congressos e publicações em revistas científicas por grupos de pesquisa de diferentes regiões do país. A avaliação do desempenho acadêmico, no entanto, tem sido feita, atualmente, nas esferas mais elevadas (tais como em instituições de fomento de pesquisa - fundações estaduais e órgãos federais - e de avaliação dos programas de pós-graduação) em função da produtividade científica de impacto internacional. Esta avaliação tem se dado por meio não apenas da análise da quantidade de artigos publicados em revistas indexadas em bases internacionais de bibliografia científica, mas também pelo fator de impacto das revistas em que os artigos são publicados. $O$ fator de impacto é calculado dividindo-se o número de vezes em que os artigos de uma revista são citados em um determinado ano, em revistas indexadas pelo Institute for Scientific Information (ISI), pelo número de trabalhos publicados pela revista nos dois anos anteriores (veja, entretanto, Coelho et al. ${ }^{1}$ e Coura $\&$ Willcox ${ }^{2}$ para críticas desta forma de avaliação). Mais recentemente, ainda, tem sido empregado o índice h (ver Hirsch ${ }^{3}$ ) para avaliação do desempenho científico individual (item disponível na Plataforma Lattes/CNPq de currículos acadêmicos), que leva em consideração diferentes fatores além do número de publicações, tais como o número total de citações recebidas pelos artigos publicados, frequência de citação por artigo publicado e quantidade de artigos de destacada importância científica (definido como artigos com número elevado de citações) publicados por um pesquisador, dentre outros indicadores.

Dada a premência de produzir ciência de impacto internacional, então, poderíamos supor que o crescimento de interesse pela área de comportamento motor no Brasil deve se manifestar não apenas em publicações nacionais, mas também em publicações com impacto no âmbito internacional. Além disso, se o desenvolvimento da área de investigação tem multiplicado os pesquisadores interessados em comportamento motor, no Brasil, poderia ser esperada uma descentralização das pesquisas de relevância internacional por pesquisadores brasileiros em anos recentes. A este respeito, foi demonstrado que a produção científica brasileira, na área da saúde e ci- ências biológicas, incluindo assim as disciplinas vinculadas à grande área de Educação Física, aumentou quatro vezes, nas últimas duas décadas, em virtude da estabilização do investimento em pesquisas e da mudança nas políticas das principais agências de financiamento ${ }^{4}$. Porém, os resultados deste estudo também revelam que as publicações científicas têm se concentrado na região sudeste do país. Este dado é consistente com a observação de maior concentração de pesquisadores nas regiões sul e sudeste, em programas de pós-graduação mais tradicionais, tais como aqueles da Universidade de São Paulo (USP), Universidade Estadual Paulista (UNESP), Universidade Estadual de Campinas (UNICAMP) e Universidade Federal de Santa Maria (UFSM), entre as décadas de 1980-19905. Mais recentemente, entre 1997 e 2000, USP, UNICAMP e Universidade Federal do Rio de Janeiro (UFRJ) têm se destacado com a publicação de $43 \%$ dos artigos indexados pelo Institute for Scientific Information (ISI) ${ }^{6}$.

A fim de formar um ponto de vista qualificado a respeito do perfil da produção científica brasileira de impacto internacional, na área de comportamento motor, neste trabalho foi feita uma quantificação de artigos publicados nesta área por pesquisadores vinculados às instituições brasileiras (1999-2008). Na sequência, apresentamos uma análise do período investigado e perspectivas de desenvolvimento para a produção científica de impacto internacional por pesquisadores brasileiros.

\section{PROCEDIMENTOS METODOLÓGICOS}

Para avaliar se tem havido de fato um aumento na produção científica nos últimos 10 anos, comparamos os 5 primeiros anos do período com os 5 anos mais recentes. A identificação dos artigos foi feita por meio dos sistemas de busca de bibliografia científica Google Acadêmico, Pubmed, Science Direct e Scopus, restringindo a busca aos seguintes termos relacionados à área de comportamento motor: motor learning (aprendizagem motora), motor/movement control (controle motor/de movimento), motor development (desenvolvimento motor), postural control (controle postural), laterality/handedness/ footedness (lateralidade/manualidade/podalidade), gait (marcha), dual task (tarefa dual) e motor coordination (coordenação motora). Foram selecionados, nesta busca, apenas os artigos com pelo menos um dos autores vinculados a uma instituição de ensino superior brasileira. Para determinação do vínculo institucional, foi considerada apenas a instituição do primeiro autor brasileiro. Foram selecionados apenas 
os periódicos com conceito "Qualis Internacional-A" e "Qualis International-B" na área de Educação Física na avaliação feita pela Coordenação de Aperfeiçoamento de Pessoal de Nível Superior (CAPES)?

\section{RESULTADOS}

Dentro dos limites estabelecidos, foram localizados 147 artigos científicos, dos quais 34 foram publicados no primeiro quinquênio analisado. Nos 5 anos finais do período, o número de publicações teve um aumento expressivo, passando para 113 artigos. Dessa forma, no primeiro quinquênio analisado foram publicados, em média, 6,8 artigos por ano em periódicos internacionais, enquanto que, no segundo quinquênio, a média aumentou para 22,6 artigos por ano. Estes números correspondem a um aumento de $332 \%$ entre os dois quinquênios. O ápice na produção científica foi atingido no ano de 2006, com um total de 30 artigos publicados.

Este conjunto de artigos foi publicado em 24 periódicos e os resultados mostram que a maior proporção de publicações ocorreu em Neuroscience Letters (15\%), enquanto que Experimental Brain Research (12\%), Gait and Posture (8\%) e Journal of Human Movement Studies (7\%) foram também veículos frequentes de publicação internacional dos estudos feitos por pesquisadores brasileiros (Tabela 1).

Tabela 1. Quantidade de publicações com participação de pesquisadores brasileiros por periódico, com discriminação por quinquênio do período 1999-2008.

\begin{tabular}{lccc}
\hline Periódico & $1^{\circ} \mathrm{Q}$ & $2^{\circ} \mathrm{Q}$ & Total \\
\hline Neuroscience Letters & 3 & 19 & 22 \\
Experimental Brain Research & 2 & 15 & 17 \\
Gait and posture & 1 & 11 & 12 \\
Journal of Human Movement Studies & 4 & 7 & 11 \\
Motor Control & 3 & 6 & 9 \\
Brain and Cognition & 3 & 6 & 9 \\
Behavioural Brain Research & 1 & 7 & 8 \\
Infant Behavior and Development & 2 & 6 & 8 \\
Journal of Biomechanics & 2 & 5 & 7 \\
Perceptual and Motor Skills & 2 & 5 & 7 \\
Human Movement Science & 2 & 4 & 6 \\
Journal of Neurophysiology & 3 & 2 & 5 \\
Journal of Motor Behavior & 1 & 3 & 4 \\
Research Quarterly for Exercise and Sport & 0 & 4 & 4 \\
Outros & 5 & 13 & 18 \\
Total & 34 & 113 & 147 \\
\hline
\end{tabular}

$1^{\circ}$ quinquênio (1999-2003), $2^{\circ}$ quinquênio (2004-2008) e total (1999-2008)
A área mais abordada nos trabalhos publicados foi controle motor (69\%), seguido por aprendizagem motora (22\%) e desenvolvimento motor (9\%), sendo que o tema mais abordado foi controle postural, com $44 \%$ dos artigos publicados dentro da área de controle motor. Com relação à autoria dos artigos, foi constatado que $90,5 \%$ dos artigos localizados estão vinculados a instituições públicas de ensino superior, com destaque para a USP (43,5\%), UFRJ (9,5\%), UNESP $(6,1 \%)$ e Universidade Federal de Minas Gerais (UFMG, 6,1\%). Os resultados completos são apresentados na Tabela 2.

Tabela 2. Quantidade de publicações por Universidade, com discriminação por quinqüênio do período 1999-2008.

\begin{tabular}{|c|c|c|c|}
\hline Universidade & $1^{\circ} \mathrm{Q}$ & $2^{\circ} \mathrm{Q}$ & Total \\
\hline USP & 19 & 45 & 64 \\
\hline UFRJ & 1 & 13 & 14 \\
\hline UNESP & 4 & 5 & 9 \\
\hline UFMG & 1 & 8 & 9 \\
\hline UFSCAR & 0 & 7 & 7 \\
\hline UNIFESP & 0 & 6 & 6 \\
\hline UFPelotas & 1 & 4 & 5 \\
\hline UFRGS & 1 & 4 & 5 \\
\hline UERJ & 3 & 1 & 4 \\
\hline UFPA & 0 & 4 & 4 \\
\hline UNICAMP & 1 & 2 & 3 \\
\hline PUC & 0 & 3 & 3 \\
\hline UNB & 1 & 1 & 2 \\
\hline UFJF & 0 & 2 & 2 \\
\hline UNIVAP & 0 & 2 & 2 \\
\hline Outros & 2 & 6 & 8 \\
\hline Total & 34 & 113 & 147 \\
\hline
\end{tabular}

USP-Universidade de São Paulo, UFRJ-Universidade Federal do Rio de Janeiro, UNESP-Universidade Estadual Paulista, UFMGUniversidade Federal de Minas Gerais, UFSCar-Universidade Federal de São Carlos, UNIFESP-Universidade Federal de São Paulo, UFPel-Universidade Federal de Pelotas, UFRGS-Universidade Federal do Rio Grande do Sul, UERJ-Universidade Estadual do Rio de Janeiro, UFPA-Universidade Federal do Pará, UNICAMP-Universidade Estadual de Campinas, PUCPontifícia Universidade Católica, UNB-Universidade de Brasília, UFJF-Universidade Federal de Juiz de Fora, UNIVAPUniversidade do Vale do Paraíba.

\section{DISCUSSÃO}

Os dados do presente estudo não apenas corroboram a impressão da expansão da área de comportamento motor no Brasil - a partir do aumento expressivo do número de publicações internacionais, nos últimos 5 anos - mas, principalmente, indicam um ganho qualitativo do trabalho de pesquisa nesta 
área. Esta conclusão está fundamentada no fato de que publicações em periódicos tradicionais da área, reconhecidos internacionalmente, enfrentam uma competição em âmbito mundial com alguns dos principais pesquisadores de centros de pesquisa estrangeiros. Isso implica uma disputa em um nível bastante elevado, em que apenas os trabalhos científicos com resultados conclusivos e possuindo maior contribuição original à produção de conhecimento são selecionados para publicação, após passar pelo rigoroso crivo de especialistas de reconhecimento internacional no tema do artigo. Tal ganho quantitativo e qualitativo representa um amadurecimento importante para a área de comportamento motor no Brasil, pois hoje, mais do que em tempos passados, a produção científica corresponde a um empreendimento coletivo a fim de empregar técnicas e conhecimentos necessários para a investigação de um problema científico ${ }^{8}$. Ao longo de um trabalho de pesquisa com potencial para chegar a uma publicação internacional, além do pesquisador principal, geralmente estão envolvidos outros pesquisadores na condição de orientador ou coautor. Com este trabalho conjunto, desenvolve-se a competência de todo um grupo de pesquisadores e estudantes, que contribui para manter o processo de ampliação da competência científica em uma área de estudo, particularmente para aquelas localmente emergentes.

Um dado contraditório com a percepção preliminar de crescimento descentralizado desta área de estudo, entretanto, é a observação de que a maior parte das publicações permanece concentrada em poucas instituições de ensino superior, mais notoriamente na USP e UFRJ. Ambas as universidades estão localizadas na região sudeste do país, local de maior concentração de pesquisadores especializados, e de maior investimento público e privado em atividades de pesquisa9. Além disso, $86 \%$ dos cursos de mestrado e $93 \%$ dos cursos de doutorado são localizados em universidades públicas e estes recebem mais de $80 \%$ dos investimentos concedidos pelo $\mathrm{CNPq}^{10}$. Esta constatação sugere que apesar do crescente interesse pela área de investigação chamada comportamento motor, com numerosa participação em congressos específicos, nos últimos anos, a produção científica qualificada para disputar visibilidade internacional ainda está restrita aos laboratórios com maior incentivo e tradição de investigação científica nesta área. Este pode representar um elemento de limitação da ampliação da produção brasileira de impacto internacional, restringindo a tendência de crescimento detectada na comparação entre os 5 primeiros e os 5 últimos anos de nossa análise. É possível que, a este respeito, os centros de pesquisa estabelecidos mais recentemente, principalmente as instituições privadas que contam com pesquisadores recém-formados, com poucos recursos financeiros e com poucos trabalhos de investigação científica ${ }^{6}$, tenham que passar por um processo de amadurecimento antes de atingir uma produtividade científica internacional consistente. Para estes grupos, a interação e trabalho em colaboração com outros laboratórios de reconhecida competência, dedicados ao estudo do comportamento motor, parece ser o caminho mais viável para se atingir um patamar mais elevado de produtividade científica de impacto internacional. Katz e Martin ${ }^{11}$ destacam que as vantagens de se trabalhar em colaboração são a partilha e a transferência de conhecimentos, habilidades e técnicas, a geração de novas perspectivas através de opiniões divergentes, o companheirismo e a ligação em uma ampla rede de contatos acadêmicos, possibilitando o aumento da visibilidade do trabalho. Em outras palavras, a descentralização da produção científica qualificada e o interesse dos membros de grupos recém-formados em procurar colaboração, revelam-se como uma necessidade para manter a atual tendência de aumento na quantidade de publicações em periódicos internacionais.

\section{REFERÊNCIAS BIBLIOGRÁFICAS}

1. Coelho PMZ, Antunes CMF, Costa HMA, Kroon EG, Sousa Lima MC, Linardi PM. The use and misuse of the "impact factor" as a parameter for evaluation of scientific publication quality: a proposal to rationalize its application. Braz J Med Biol Res 2003; 36(12):1605-1612.

2. Coura JR, Willcox LCB. Impact factor, scientific production and quality of Brazilian medical journals. Mem Inst Oswaldo Cruz 2003;98(3):293-297

3. Hirsch JE. An index to quantify an individual's scientific research output. Proc Natl Acad Sci USA 2005;02(46):16569-16572.

4. Correa UC, Perotti Jr. A, Pellegrini AM. Tendências dos estudos de aprendizagem e desenvolvimento motor na literatura brasileira em Educação Física. Motriz 1995;1(2):92-101.

5. Zorzetto R, Razzouk D, Dubugras MTB, et al. The scientific production in health and biological sciences of the top 20 Brazilian universities. Braz J Med Biol Res 2006;39(12):1513-1520.

6. De Meis L, Velloso A, Lannes D, Carmo MS, De Meis C. The growing competition in Brazilian science: rites of passage, stress and burnout. Braz J Med Biol Res 2003; 36(9):1135-1141 
7. Coordenação de Aperfeiçoamento de Pessoal de Nível Superior (CAPES). Qualificação de periódicos, anais, revistas e jornais (Qualis); disponível em: $<$ http://qualis. capes.gov.br/webqualis/> [2009 março 06].

8. Velloso A, Lannes D, De Meis L. Concentration of science in Brazilian governmental universities. Scientometrics 2004;61(2):207-220.

9. Gregolin JAR, Hoffmann WAM, De Faria LIL, Quoniam L, Queyras J, Fratucci LFA, et al. Análise da produção científica a partir de indicadores bibliométricos. In: Landi FR, editor. Indicadores de ciência, tecnologia e inovação em São Paulo. São Paulo: FAPESP; 2004. p.5-43.

10. Leta J, Lannes D, De Meis L. Human resources and scientific productivity in Brazil. Scientometrics 1998;41(3):313-324.
11. Katz JSK, Martin BR. What is research collaboration? Research Policy 1997;26(1):1-18.

\section{Endereço para correspondência}

Universidade de São Paulo

Escola de Educação Física e Esporte

Av. Prof. Mello Moraes, 65

05508-900 - São Paulo, Brasil

E-mail: lateixei@pq.cnpq.br 